\title{
Clinical metagenomic identification of Balamuthia mandrillaris encephalitis and assembly of the draft genome: the continuing case for reference genome sequencing
}

Alexander L. Greninger ${ }^{1,2+}$, Kevin Messacar ${ }^{3+}$, Thelma Dunnebacke ${ }^{4}$, Samia N. Naccache ${ }^{1,2}$, Scot Federman ${ }^{1,2}$, Jerome Bouquet ${ }^{1,2}$, David Mirsky ${ }^{3}$, Yosuke Nomura ${ }^{3}$, Shigeo Yagi ${ }^{4}$, Carol Glaser ${ }^{5}$, Michael Vollmer ${ }^{6}$, Craig A. Press ${ }^{3}$, Bette K. Kleinschmidt-DeMasters ${ }^{3}$, Samuel R. Dominguez ${ }^{3}$ and Charles Y. Chiu ${ }^{1,2,7^{*}}$

\begin{abstract}
Background: Primary amoebic meningoencephalitis (PAM) is a rare, often lethal, cause of encephalitis, for which early diagnosis and prompt initiation of combination antimicrobials may improve clinical outcomes.

Methods: In this study, we sequenced a full draft assembly of the Balamuthia mandrillaris genome (44.2 Mb in size) from a rare survivor of PAM, and recovered the mitochondrial genome from six additional Balamuthia strains. We also used unbiased metagenomic next-generation sequencing (NGS) and SURPI bioinformatics analysis to diagnose an ultimately fatal case of Balamuthia mandrillaris encephalitis in a 15-year-old girl.

Results and Discussion: Comparative analysis of the mitochondrial genome and high-copy number genes from six additional Balamuthia mandrillaris strains demonstrated remarkable sequence variation, and the closest Balamuthia homologs corresponded to other amoebae, hydroids, algae, slime molds, and peat moss. Real-time NGS testing of hospital day 6 CSF and brain biopsy samples detected Balamuthia on the basis of high-quality hits to $16 \mathrm{~S}$ and $18 \mathrm{~S}$ ribosomal RNA sequences present in the National Center for Biotechnology Information (NCBI) nt reference database. The presumptive diagnosis of PAM by visualization of amoebae on brain biopsy histopathology and NGS analysis was subsequently confirmed at the US Centers for Disease Control and Prevention (CDC) using a Balamuthia-specific PCR assay. Retrospective analysis of a day 1 CSF sample revealed that more timely identification of Balamuthia by metagenomic NGS, potentially resulting in a better clinical outcome, would have required availability of the complete genome sequence.

Conclusions: These results underscore the diverse evolutionary origins of Balamuthia mandrillaris, provide new targets for diagnostic assay development, and will facilitate further investigations of the biology and pathogenesis of this eukaryotic pathogen. The failure to identify PAM from a day 1 sample without a fully sequenced Balamuthia genome in the database highlights the critical importance of whole-genome reference sequences for microbial detection by metagenomic NGS.
\end{abstract}

\footnotetext{
* Correspondence: charles.chiu@ucsf.edu

${ }^{\dagger}$ Equal contributors

${ }^{1}$ Department of Laboratory Medicine, University of California, 185 Berry

Street, Box 0134, San Francisco, CA 94107, USA

${ }^{2}$ UCSF-Abbott Viral Diagnostics and Discovery Center, San Francisco, CA

91407, USA

Full list of author information is available at the end of the article
}

C Biomed Central (c) 2015 Greninger et al. Open Access This article is distributed under the terms of the Creative Commons Attribution 4.0 International License (http://creativecommons.org/licenses/by/4.0/), which permits unrestricted use, distribution, and reproduction in any medium, provided you give appropriate credit to the original author(s) and the source, provide a link to the Creative Commons license, and indicate if changes were made. The Creative Commons Public Domain Dedication waiver (http://creativecommons.org/publicdomain/zero/1.0/) applies to the data made available in this article, unless otherwise stated. 


\section{Background}

Balamuthia mandrillaris is a free-living amoeba that is a rare, almost uniformly lethal, cause of primary amoebic meningoencephalitis (PAM) in humans [1]. Originally isolated from the brain of a baboon at the San Diego Zoo in 1986, Balamuthia mandrillaris has since been reported in over 100 cases of PAM worldwide [2-4], and amoebae associated with fatal encephalitis in a child have been cultured directly from soil [5]. The vast majority of cases are fatal, although there are a few published case reports of patients surviving Balamuthia encephalitis after receiving combination antimicrobial therapy and in vitro data supporting the potential efficacy of several antimicrobial agents [3, 6-10]. Despite the availability of validated RT-PCR assays for the detection of freeliving amoebae $[11,12]$, PAM is not often clinically suspected and the diagnosis is most commonly made around the time of death or postmortem on brain biopsy [13, 14].

Our lab has demonstrated the capacity of metagenomic next-generation sequencing (NGS) to provide clinically actionable information in a number of acute infectious diseases, most notably encephalitis $[15,16]$. This approach enables the rapid and simultaneous detection of viruses, bacteria, and eukaryotic parasites in clinical samples [17]. Encephalitis is a critical illness with a broad differential, for which unbiased diagnostic tools such as metagenomic NGS can make a significant impact [18]. However, the utility of diagnostic NGS is highly dependent on the breadth and quality of databases that contain wholegenome sequence information of reference strains needed for alignment [19].

In this study, we describe the first draft genome sequence of a strain of Balamuthia mandrillaris from a rare survivor of PAM and comparative sequence analysis of six additional mitochondrial genomes. We also demonstrate the ability of metagenomic NGS to rapidly detect Balamuthia mandrillaris from the cerebrospinal fluid (CSF) of a critically ill 15-year-old girl, and highlight the importance of genomic reference sequences by retrospective analysis of a hospital day (HD) 1 sample.

\section{Methods \\ Ethics}

Written informed consent was obtained from the parents of the patient for analysis of her clinical samples, in accordance with a study approved by the Colorado Multiple Institutional Review Board (IRB). Written informed consent was also obtained from the parents for publication of this research. Coded samples from the patient were analyzed for pathogens by NGS under study protocols approved by the University of California, San Francisco IRB.

\section{Metagenomic sequencing of CSF and brain biopsy}

Total nucleic acid was extracted from $200 \mu \mathrm{L}$ of CSF using the Qiagen EZ1 Viral kit. Half of the nucleic acid from CSF was treated with Turbo DNase (Ambion). Total RNA was extracted from $2 \mathrm{~mm}^{3}$ brain biopsy tissue using the Direct-zol RNA MiniPrep Kit (Zymo Research), followed by mRNA purification using the Oligotex mRNA Mini Kit (Qiagen). Total RNA from CSF and mRNA from brain biopsy were reverse-transcribed using random hexamers and randomly amplified as previously described [20]. The resulting double-stranded cDNA or extracted DNA from CSF (the fraction not treated with Turbo DNase) was used as input into Nextera XT, following the manufacturer's protocol except with reagent volumes cut in half for each step in the protocol. After 14-18 cycles of PCR amplification, barcoded libraries were cleaned using Ampure XP beads, quantitated on the BioAnalyzer (Agilent), and run on the Illumina MiSeq $(1 \times 160$ bp run). Metagenomic NGS data were analyzed for pathogens via SURPI using NCBI nt/nr databases from June 2014 [17].

A rapid taxonomic classification algorithm based on the lowest common ancestor was incorporated into SURPI, as previously described [20], and used to assign viral, bacterial, and non-chordate eukaryotic NGS reads to the species, genus, or family level. For the SNAP nucleotide aligner [21], an edit distance cutoff of 12 was used for viral reads [17], but adjusted to a more stringent edit distance of 6 for bacterial and non-chordate eukaryotic reads to increase specificity.

Rigorous measures were taken to prevent laboratory contamination and cross-contamination in this study. This included: (1) library preparation and sequencing of day 1 clinical samples, day 6 samples, and Balamuthia cultures on different weeks (with at least three sequencing runs in between to minimize carryover); (2) unidirectional laboratory workflow, with separate rooms for the pre-PCR and post-PCR steps; (3) cleanup of the work area prior to and after specimen processing with $10 \%$ sodium hypochlorite; (4) use of disposable gloves and biohazard pouches that are frequently changed; and (5) dual-index barcoding of multiplexed clinical samples within individual runs. A negative control clinical CSF sample from an unrelated patient was processed in parallel with the case patient's CSF and brain biopsy samples at day 1 , and was negative for any reads aligning to Balamuthia. Furthermore, the nine Balamuthia DNA library sequences observed in the patient's day 1 CSF were unique and did not overlap any of the 926 DNA library sequences in day 6 CSF, excluding the possibility of amplicon contamination.

\section{Propagation of Balamuthia mandrillaris in culture}

Trypsin-treated cultures from Vero or BHK (baby hamster kidney) cell monolayers and Balamuthia mandrillaris 
amoebas were placed into T25 culture flasks in Dulbecco's Modified Eagle Medium (DMEM) plus $10 \%$ bovine serum, $1 \%$ of the antibiotics $100 \mathrm{U} / \mathrm{mL}$ penicillin, $0.1 \mathrm{mg} /$ $\mathrm{mL}$ streptomycin, and $0.25 \mu \mathrm{g} / \mathrm{mL}$ fungizone, and incubated at $37{ }^{\circ} \mathrm{C}$ for 7 to 10 days plus 2 days at room temperature until the underlying cell sheet was completely destroyed and only actively dividing amebae were seen floating and/or attached to the surface. Attached amoebas were freed by gently tapping the side of the flask or putting the flask on a bed of ice for $20 \mathrm{~min}$. The amoebas were concentrated by centrifugation for $5 \mathrm{~min}$. After removal of supernatant, the amoeba pellet was washed by addition of PBS, centrifuged again, and then placed in a flask with Bacto-casitone axenic medium and allowed to grow for another 7-10 days, after which the amoebas were concentrated again, washed, and placed into fresh axenic medium [22]. After a final centrifugation step, the amebae were collected, washed $3 \times$ in PBS, pelleted, and stored at $-80{ }^{\circ} \mathrm{C}$.

\section{Sequencing and annotation of cultured Balamuthia mandrillaris 2046 strain}

DNA from individual strains of Balamuthia mandrillaris was extracted using the Qiagen EZ1 Tissue Kit and used as input for the Nextera Mate Pair Kit (Illumina) and Nextera XT Kit (Illumina), following the manufacturer's instructions. Mate-pair libraries were sequenced on an Illumina MiSeq $(2 \times 80 \mathrm{nt}$ and $2 \times 300 \mathrm{nt}$ runs), while the Nextera XT library was sequenced on an Illumina HiSeq $(2 \times 250$ bp paired-end sequencing) (Table 2). Mate-pair reads from run MP1 were adapter-trimmed with NxTrim [23], and the mitochondrial genome of strain 2046 and high-copy number contigs were assembled using SPAdes v3.5 $[24,25]$. The average insert size of the mate-pair library was 2,187 nucleotides. Prediction of tRNA and rRNA genes was performed using tRNAscan-SE and RNAmmer v1.2, respectively [26, 27]. ORFs were predicted in translation code 4 with the Glimmer gene predictor, and all predicted ORF sequences were confirmed using BLASTx and HHPred [28, 29].

Reads from runs MP2 and MP3 were mate-pair adapter-trimmed using NxTrim, while reads from all runs were quality-filtered (q30) and adapter-trimmed using cutadapt [30]. Reads that aligned to the Balamuthia mitochondrial genome and golden hamster (Mesocricetus auratus) were identified using SNAP [21] and removed prior to de novo assembly using platanus [31]. Any scaffold of length less than $500 \mathrm{bp}$ along with 62 scaffolds that aligned to Mesocricetus auratus, Chlorocebus sabaeus, Waddlia chondrophila, and Enterobacteria phage phiX174 (all likely deriving from cell culture contamination) were removed.

\section{Rps3 PCR confirmation}

Genomic DNA was PCR-amplified using $0.5 \mu \mathrm{M}$ final concentration of primers rps3-F (5'-CTGYTCGATTTT CGAAAAATAAAGTAG-3') and rps3-R (5'-TGAAAGAAGAACATTTAGATCACGACT-3') using 2X iProof HF Master Mix (Bio-Rad) in $20 \mu \mathrm{L}$ total volume. Conditions were $95^{\circ} \mathrm{C} \times 2 \mathrm{~min}$, followed by 35 cycles of $95^{\circ} \mathrm{C} \times$ $30 \mathrm{~s}, 52{ }^{\circ} \mathrm{C} \times 30 \mathrm{~s}, 72{ }^{\circ} \mathrm{C} \times 40 \mathrm{~s}$, and a final incubation at $72{ }^{\circ} \mathrm{C} \times 2 \mathrm{~min}$. PCR amplicons were visualized by $3 \%$ agarose gel electrophoresis.

\section{Data access}

The Balamuthia mandrillaris mitochondrial genomes have been deposited in NCBI under the following accession numbers: 2046 axenic (KP888565), 2046-1 (KT175740), V451 (KT030670), OK1 (KT030671), RP-5 (KT030672), SAM (KT030673), V188-axenic (KT175738), V188-frozen stock (KT175739), and V039 (KT175741). The Balamuthia mandrillaris scaffolds have been deposited in the NCBI Whole Genome Shotgun (WGS) database under accession number LEOU00000000. Metagenomic NGS data corresponding to the patient's brain biopsy and CSF fluid, as well as corresponding to a negative control CSF sample from an unrelated patient, have been submitted to the NCBI Sequence Read Archive (SRA) under accession number SRP064607. NGS reads were filtered for exclusion of human sequences using Bowtie2 high-sensitivity local alignment to the human hg38 reference database at default parameters followed by BLASTn alignment to all primate sequences in NCBI nt at an e-value cutoff of $10^{-8}$ prior to deposition into the NCBI SRA database.

\section{Results}

\section{First mitochondrial genome of Balamuthia mandrillaris}

We cultured Balamuthia mandrillaris strain 2046 in axenic media from a brain biopsy corresponding to a rare survivor of PAM (Vollmer and Glaser, manuscript in review). Sequencing of DNA extracted from the axenic culture generated 3.8 million 75 base pair (bp) mate-pair reads with an average insert size of 2,187 bp. De novo assembly yielded a circular mitochondrial genome of 41,656 bases that was comprised of $64.8 \%$ AT at $2,082 \times$ coverage (Fig. 1a). The overall size and AT content of the Balamuthia mandrillaris mitochondrial genome was closer to that of Acanthamoeba castellani (41,591 bp, $70.6 \%$ AT) [32] than Naegleria fowleri (49,531 bp, $74.8 \%$ AT) [33], although overall average nucleotide identity with Balamuthia was found to be low for both amoebae (approximately $68 \%$ ).

The Balamuthia mandrillaris 2046 mitochondrial genome contained two ribosomal RNA (rRNA) genes, 18 transfer RNA (tRNA) genes, and 38 coding sequences, with five of those being hypothetical proteins. The organization of the mitochondrial genome retained 


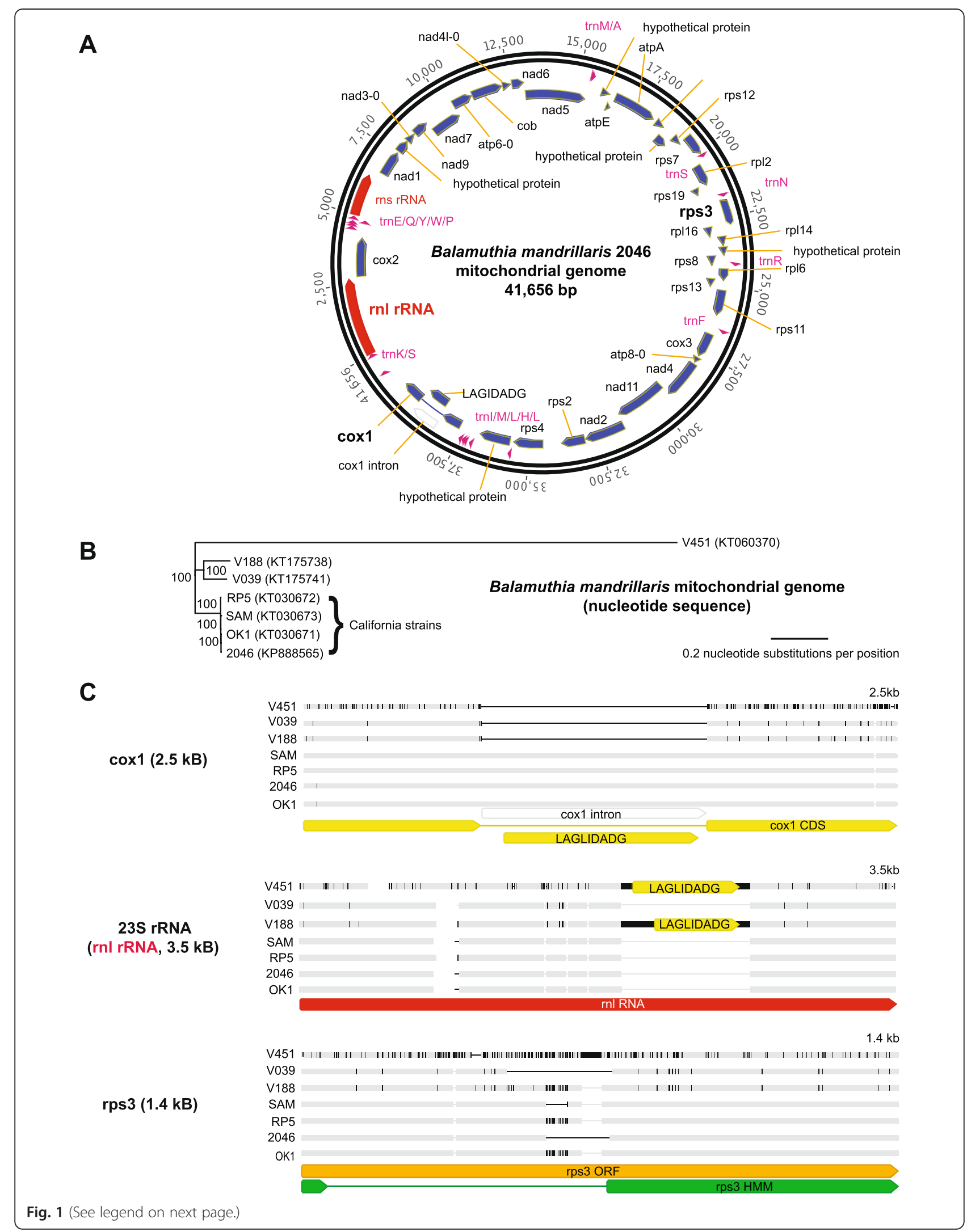


(See figure on previous page.)

Fig. 1 Sequencing and comparative phylogenetic analysis of the mitochondrial genome of Balamuthia mandrillaris. a Balamuthia mandrillaris 2046 mitochondrial genome. Annotation of the 41,656 bp genome was performed using RNAmmer, tRNAscan-SE, and Glimmer gene predictor, with all ORFs manually verified using BLASTx alignment. b Phylogenetic analysis of seven newly sequenced mitochondrial genomes from different strains of Balamuthia mandrillaris. An outgroup (for example, Acanthamoeba castellani) is not shown given the lack of gene synteny. Branch lengths are drawn proportionally to the number of nucleotide substitutions per position, and support values are shown for each node. $\mathbf{c}$ Differences in individual gene features (cox1, 23S rRNA, and rps3), among the 7 mitochondrial genomes, as detailed in the text. The mitochondrial cox1, 23S rRNA / rnl RNA, and rps3 genes are highlighted in boldface

several syntenic blocks with the Acanthamoeba castellani genome, including tnad3-9-7-atp6 and rpl11-rps12rps7-rpl2-rps19-rps3-rpl16-rpl14. However, many other features of the genome were unique, such as the order of the remaining coding blocks, the lack of a combined cox1/cox2 gene, as present in Acanthamoeba castellani [32], and the lack of intron splicing in 23S rRNA. The Balamuthia mandrillaris mitochondrial cox1 gene was interrupted by a LAGLIDADG endonuclease open reading frame (ORF) containing a group I intron, as has been reported for a wide variety of other eukaryotic species $[34,35]$. The putative rps3 gene was encoded within a 1,290 bp ORF that, when translated, aligned by hidden Markov model (HMM) analysis to rps3 proteins from Escherichia coli (PDB 4TP8/4U26) and Thermus thermophilus (PDB 4RB5/4W2F), and only in base positions 166 and 583-1290 of the ORF. This finding was consistent with the presence of a putative $>500$ bp intron in the Balamuthia mandrillaris rps3 gene that to date has only been described to in plants [36, 37]. Alternatively, the ORF was also found to encode a putative tRNA (Asn) such that the $5^{\prime}$ end of the ORF could represent a large intergenic sequence.

Mitochondrial genome diversity of Balamuthia mandrillaris To investigate the extent of sequence diversity in Balamuthia mandrillaris, we sequenced the mitochondrial genomes from six additional Balamuthia strains obtained from the California Department of Public Health and the American Tissue Culture Collection (Table 1). The seven total circular mitochondrial genomes averaged 41,526 bp in size (range, 39,996 to 42,217 bp), and shared pairwise nucleotide identities in the range of $82.6 \%$ to $99.8 \%$. The phylogeny revealed the presence of at least three separate lineages of Balamuthia mandrillaris, with all four strains from human cases in California clustering together in a single clade (Table 1; Fig. 1b). Consistent with a previous report [38], we found that the mitochondrial genome of strain V451 was the most divergent among tested strains, and possessed an additional 1,149 bp ORF downstream of the cox1 gene that did not align significantly to any sequence in the NCBI nt or nr reference database. Of note, our V039 mitochondrial genome assembly showed > $99.9 \%$ identity to a recently deposited PacBio / Illumina assembly of the V039 mitochondrial genome. The only difference between the two mitochondrial genomes was a 102-bp insertion that was located in the unique rps3 locus [39].

Putative introns constituted the major source of variation among the mitochondrial genomes (Fig. 1c). Four strains out of seven, including strain 2046 from the rare survivor of Balamuthia infection, contained a 975 bp LAGLIDADG-containing intron in the cox 1 gene, whereas no such intron was present in the remaining three strains. Two of the remaining three strains, strains V451 and V188, instead had an approximately 790 bp insert in the $23 \mathrm{~S}$ rRNA gene (Fig. 1a, 'rnl RNA') that contained a 530 bp or 666 bp LAGLIDADG-containing ORF,

Table 1 Strains used in this study

\begin{tabular}{|c|c|c|c|c|}
\hline Strain & Date & Location & Note & Citation \\
\hline $\mathrm{SAM}^{\mathrm{a}}$ & Spring 2001 & California & 3-year-old girl, cultured on Vero cells & Bakardjiev A et al., 2002 [55] \\
\hline $\mathrm{RP}-5^{\mathrm{a}}$ & Spring 2001 & California & Environmental sample associated with SAm, cultured on Vero cells & Schuster FL et al., 2003 [5] \\
\hline $\mathrm{OK} 1^{\mathrm{a}}$ & 2002 & California & Environmental sample in NorCal unrelated to SAm, cultured on Vero cells & Dunnebacke TH et al., 2004 [56] \\
\hline V039b & 1990 & California & $\begin{array}{l}\text { Type strain isolated from pregnant mandrill at San Diego Zoo, cultured } \\
\text { on Vero cells }\end{array}$ & Visvesvara GS et al., 1990 [4] \\
\hline $\mathrm{V} 188^{\mathrm{a}}$ & 1989 & Georgia & 59-year-old man, amoeba isolated from brain/skin lesion, cultured on Vero cells & Gordon SM et al., 1992 [57] \\
\hline $\mathrm{V} 451^{\mathrm{a}}$ & - & New York & 6-year-old girl, cultured on Vero cells & Yang XH et al., 2001 [58] \\
\hline $2046^{a}$ & March 2010 & California & 26-year-old man, survivor, cultured on Vero cells & Vollmer and Glaser, in review \\
\hline CSF108 ${ }^{c}$ & May 2015 & Colorado & 15-year-old girl, direct metagenomic detection from CSF & \\
\hline
\end{tabular}

${ }^{\mathrm{a}}$ Strain provided by Dr. Dunnebacke at the California Department of Public Health

${ }^{\mathrm{b}}$ Strain obtained from ATCC (V39)

'Strain obtained from recent clinical case in Colorado 
respectively, and that coded for a putative endonuclease. The LAGLIGDADG-containing endonucleases in the two strains shared $84 \%$ amino acid pairwise identity with each other, but approximately $50 \%$ amino acid identity to a corresponding LAGLIGDADG-containing endonuclease in the 23S rRNA gene of Acanthamoeba castellani, and $<12 \%$ amino acid identity to the LADGLIDADG-containing cox1 introns in the four other Balamuthia strains. The final remaining strain, ATCC-V039, lacked an intron in either the cox1 or 23S rRNA gene.

The ORF containing the rps3 gene, found to contain a possible rps3 intron / intergenic region by analysis of the strain 2046 mitochondrial genome, varied in length among the seven sequenced mitochondrial genomes from $1,290 \mathrm{bp}$ to $1,425 \mathrm{bp}$. Notably, the length of the putative intron / intergenic region accounted for all of the differences in overall length of the rps3 gene. Confirmatory PCR and sequencing of this locus using conserved outside primers revealed that each strain tested had a unique length and sequence (Fig. 2), raising the possibility of targeting this region for Balamuthia mandrillaris strain detection and genotyping.

\section{First draft genome of Balamuthia mandrillaris}

Because of the high-copy number of mitochondrial sequences in the Balamuthia sequencing run, as noted previously for Naegleria fowleri [33], we performed an additional NGS run of 14.1 million 250 bp single-end reads, and computationally subtracted reads aligning to the mitochondrial genome. Assembly of the remaining 4.4 million high-quality reads yielded 31,194 contiguous sequences (contigs) with an N50 of 3,411 bp. Scaffolding and gap closure using an additional 57.4 million NGS reads and computational removal of exogenous sequence contaminants yielded a final assembly of approximately $44.3 \mathrm{Mb}$ comprising 14,699 scaffolds with an N50 of 19,012 bp (Table 2). Direct BLASTn alignment of the scaffolds to the National Center for Biotechnology Information (NCBI) nt database revealed that the most common organism aligning to Balamuthia mandrillaris was Mus musculus (house mouse) $(2,067 / 14,699=14.1 \%$ of scaffolds), nearly entirely due to low-complexity sequences, followed by high-significance hits to Acanthamoeba castellani $(627 / 14,699=4.3 \%$ of scaffolds $)$.

Phylogentic analysis of individual genes from the Balamuthia mitochondrial genome revealed the presence of significant diversity across all kingdoms of life (Fig. 3). The 18S-28S rRNA locus in the Balamuthia mitochondrial genome corresponded to a $12.5 \mathrm{kB}$ contig sequenced at high coverage ( $>400 \times$ over rRNA regions). The previously sequenced $18 \mathrm{~S}$ gene $(2,017 \mathrm{bp})$ demonstrated $99.5 \%$ identity to existing Balamuthia mandrillaris 18S rRNA sequences in the NCBI nt database, while the $28 \mathrm{~S}$ gene (4,999 bp) had homology across multiple diverse species, with only $68.5 \%$ pairwise identity to its closest phylogenetic relative, Acanthamoeba castellani (Fig. 3b). From the nuclear genome, one high-copy contig contained a truncated 5,250 nucleotide ORF exhibiting only $33 \%$ amino acid identity to Rhizopus delemar (pin mold), and harboring elements consistent with a retrotransposon [40], including an RNAse HI from Ty3/ Gypsy family retroelements, a reverse transcriptase, a chromodomain, and a retropepsin. Two high-copy, approximately $1,600 \mathrm{bp}$ ORFs that failed to match any sequence by BLASTx alignment to the NCBI nr protein database were found to align significantly to Escherichia coli site-specific recombinase by remote homology HMM analysis.

\section{A case of Balamuthia encephalitis diagnosed by metagenomic NGS}

Concurrent with assembly of the Balamuthia genome, metagenomic NGS was performed to investigate a case of meningoencephalitis in a 15-year-old girl with insulin-

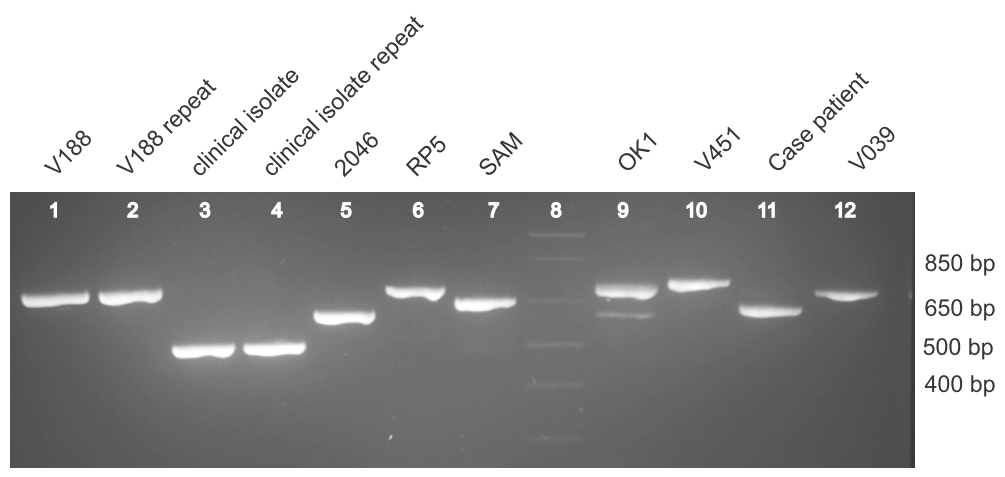

Fig. 2 PCR amplification of the Balamuthia rps3 mitochondrial gene. The variable length of the rps3 intron among eight different Balamuthia strains (seven newly sequenced mitochondrial genomes and the case patient) suggests that this gene may be an attractive target for development of a molecular genotyping assay. Lane 8 corresponds to the DNA ladder (faint appearance), while lanes 3 and 4 correspond to an additional clinical Balamuthia isolate whose mitochondrial genome was not sequenced 
Table 2 Sequencing runs and genome assembly details

\begin{tabular}{|c|c|c|c|c|}
\hline Sequencing run & Raw reads & Filtered reads & Read length & Library prep \\
\hline$M P 1^{a}$ & 3845734 & N/A & $2 \times 80 \mathrm{bp}$ & Nextera Mate-Pair \\
\hline$M P 2^{\mathrm{a}}$ & 31223454 & 12095792 & $2 \times 80 \mathrm{bp}$ & Nextera Mate-Pair \\
\hline $\mathrm{MP}^{\mathrm{a}}$ & 14121900 & 4397510 & $2 \times 250 \mathrm{bp}$ & Nextera Mate-Pair \\
\hline$P E 1^{b}$ & 213604902 & 29314946 & $2 \times 250 \mathrm{bp}$ & Nextera XT \\
\hline Genome Assembly $^{c}$ & Contigs & N50 (bp) & Assembly Size & \\
\hline Mitochondria & 1 & 41,656 & 41,656 & \\
\hline Contigs & 31,194 & 3,411 & $48,938,887$ & \\
\hline Scaffolds & 26,811 & 19,415 & $49,120,517$ & \\
\hline Gap-fill & 26,811 & 19,012 & $48,939,625$ & \\
\hline Final assembly (minus $<500 \mathrm{bp}$, contaminants) & 14,699 & 26,144 & $44,270,879$ & \\
\hline
\end{tabular}

${ }^{a}$ Three runs of a Nextera mate-pair library of Balamuthia strain 2046 from axenic culture sequenced on an Illumina MiSeq

${ }^{b}$ Nextera XT paired-end library of strain 2046 sequenced on an Illumina HiSeq

${ }^{\mathrm{c}}$ Assembly of mitochondrial genome from MP1 library, of whole-genome from all four libraries

dependent diabetes mellitus and celiac disease. The patient initially presented to a community emergency room with 7 days of progressive symptoms including right arm weakness, headache, vomiting, ataxia, and confusion. Her diabetes was well controlled with an insulin pump, and she did not take any additional medications. Exposure history was significant for contact with alpacas at a family farm and swimming in a freshwater pond 9 months prior. She had no international travel, sick contacts, or insect bites. She was given $10 \mathrm{mg}$ dexamethasone with symptomatic improvement in her headaches, but was subsequently transferred to a tertiary care children's hospital after a computed tomography (CT) scan revealed left occipital and frontal hypodensities.

On HD 1, peripheral white blood count was $11.6 \times 10^{3}$ cells $/ \mu \mathrm{L}$ (89\% neutrophils, $6 \%$ lymphocytes, $4 \%$ monocytes), erythrocyte sedimentation rate was $13 \mathrm{~mm} / \mathrm{h}$ (normal range, $0-20 \mathrm{~mm} / \mathrm{h}$ ), C-reactive protein was $3 \mathrm{mg} / \mathrm{dL}$ (normal range, $0-1 \mathrm{mg} / \mathrm{dL}$ ), and procalcitonin $0.05 \mathrm{ng} / \mathrm{mL}$ (normal range, $0-0.5 \mathrm{ng} / \mathrm{mL}$ ). CSF analysis demonstrated 377 leukocytes/ $\mu \mathrm{L}$ ( $2 \%$ neutrophils, $53 \%$ lymphocytes, $39 \%$ monocytes, and $6 \%$ eosinophils), glucose of $122 \mathrm{mg} / \mathrm{dL}$ (normal range, $40-75 \mathrm{mg} / \mathrm{dL}$ ), and protein of $59 \mathrm{mg} / \mathrm{dL}$ (normal range, $12-60 \mathrm{mg} / \mathrm{dL}$ ). Viral polymerase chain reaction (PCR) testing for herpes simplex virus (HSV) and bacterial cultures were negative. Magnetic resonance imaging (MRI) scan of the brain on HD 1 showed hemorrhagic lesions with surrounding edema in the superior left frontal lobe and left occipital lobe with a small focus of edema in the right cerebellum (Fig. 4a).

Given the patient's autoimmune predisposition and hemorrhagic appearance of the brain lesions, acute hemorrhagic leukoencephalitis, an autoimmune disease, was initially suspected and intravenous methylprenisolone (1,000 mg daily) was given HD 2-5. The patient clinically deteriorated with worsening headache, increasing weakness, and altered mental status on HD 5. Repeat MRI on HD 5 demonstrated enlargement of the previous hemorrhagic lesions with interval development of multiple rim-enhancing lesions (Fig. 4b). Steroids were discontinued and broad-spectrum antimicrobial therapy with vancomycin, cefotaxime, metronidazole, amphotericin B, voriconazole, and acyclovir was initiated. On HD 6, she underwent craniotomy for brain biopsy, revealing partially necrotic white matter, and had an external ventricular drain placed. CSF wet mount and gram stain, bacterial and fungal cultures, PCR testing for HSV and varicella-zoster virus (VZV), and oligoclonal bands were negative. Pathology of the brain biopsy sample showed a hemorrhagic necrotizing process with neutrophils, tissue necrosis, vasculitis, and numerous amoebae. She was additionally started on azithromycin, sulfadizine, pentamidine, and flucytosine on HD 7. On HD 8, she developed intracranial hypertension, cardiac arrest and died. Miltefosine had been requested and was en route from the $\operatorname{CDC}[3,9,41]$; however this medication did not arrive in time to administer before the patient died. Parallel metagenomic NGS testing of CSF and brain biopsy samples around the time of death confirmed the presence of sequences from Balamuthia mandrillaris (see below). Autopsy was not performed according to the wishes of the family. Subsequent clinical laboratory testing of the brain biopsy sample at the US Centers for Disease Control and Prevention $(\mathrm{CDC})$, using a specific PCR targeting the RNAse $\mathrm{P}$ gene [42], confirmed the diagnosis of Balamuthia mandrillaris encephalitis. 
A

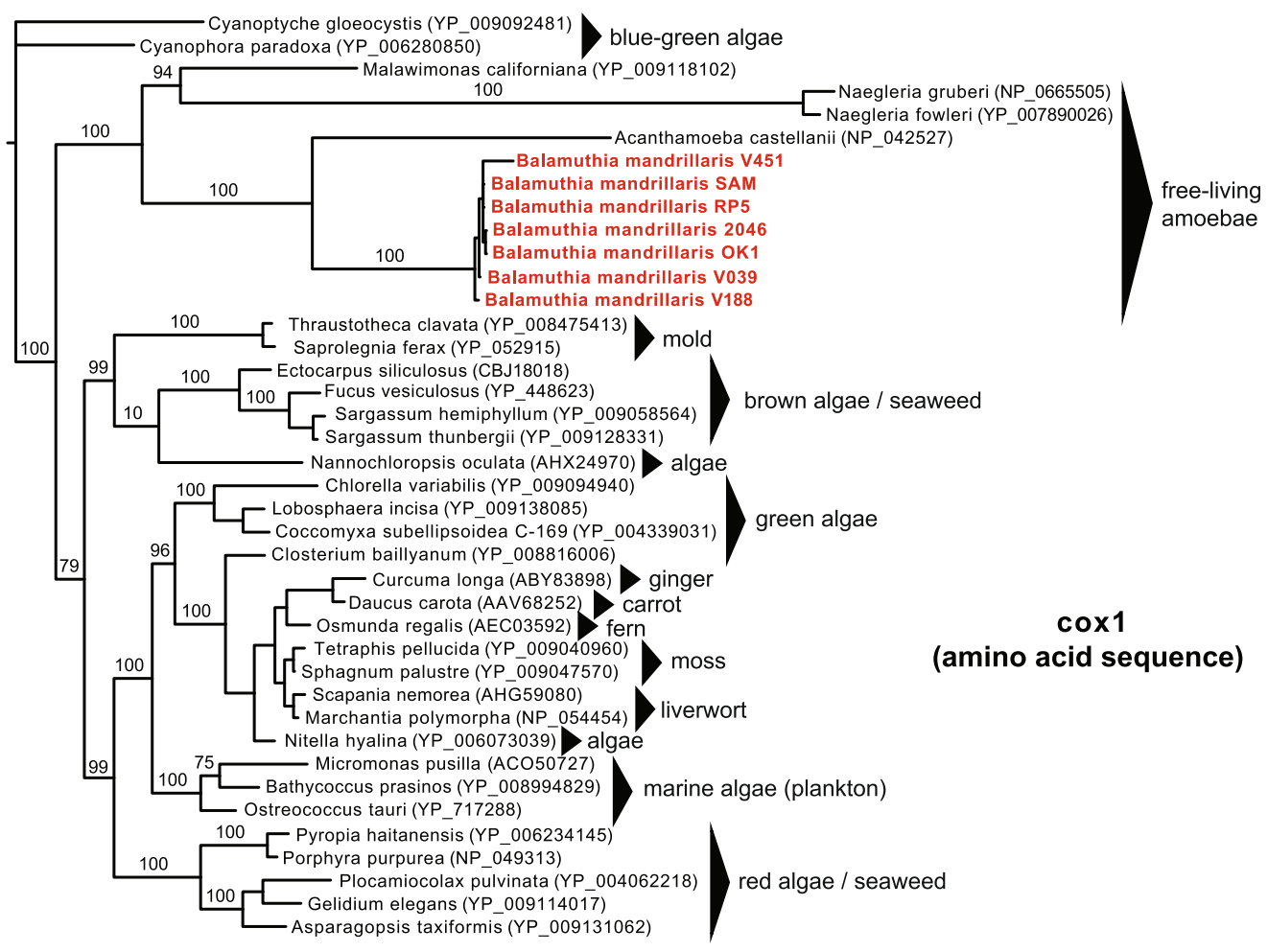

B

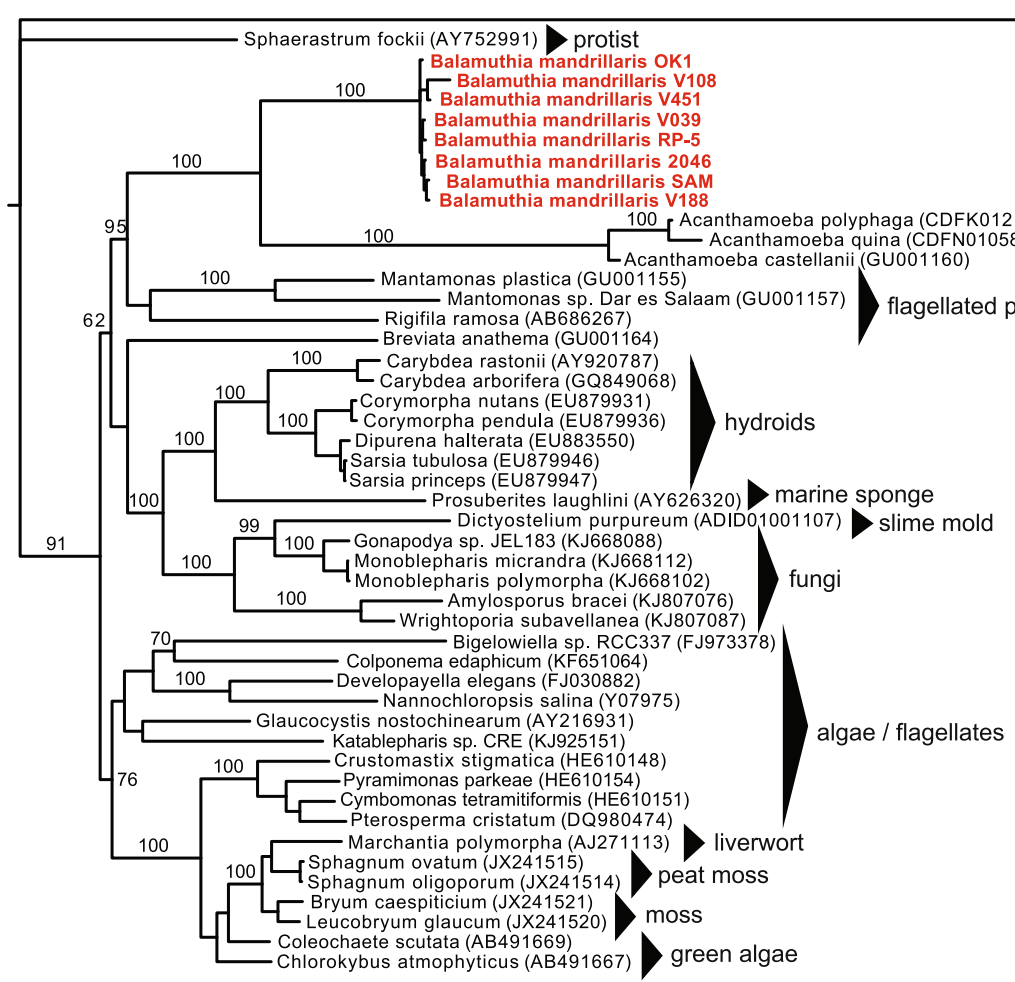

Naegleria gruberi (NC_010189)

free-living

amoebae

100 Acanthamoeba polyphaga (CDFK01213195)

)

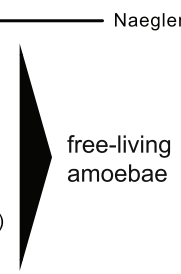

Mantamonas plastica (GU001155) Rigifil Mantomonas sp. Dar es Salaam (GU001157) flagellated protists Breviata anathema (GU001164)

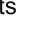


(See figure on previous page.)

Fig. 3 Phylogenetic trees of the mitochondrial cox 1 protein and $28 \mathrm{~S}$ rRNA gene reveal the close phylogenetic relationship between Balamuthia and Acanthamoeba. a Phylogeny of seven Balamuthia cox1 amino acid sequences along with the top complete sequence hits in NCBI nr ranked by BLASTp E-score. $\mathbf{b}$ Phylogeny of seven Balamuthia $23 \mathrm{~S}$ rRNA nucleotide sequences along with the top complete sequence hits in NCBI nt ranked by BLASTn E-score. Sequences were aligned using MUSCLE and a phylogenetic tree constructed using MrBayes. Branch lengths are drawn proportionally to the number of nucleotide substitutions per position, and support values are shown for each node

\section{Identification of Balamuthia in CSF and brain biopsy material}

Metagenomic NGS and SURPI bioinformatics analysis were used to analyze the patient's HD 6 CSF and brain biopsy for potential pathogens. Analysis of the viral portion of RNA or DNA derived reads revealed only phages or misannotated sequences (Additional file 1: Table S1), while most of the bacterial reads mapped to common skin / environmental contaminants such as Propionibacterium and Staphylococcaceae in the HD 6 CSF RNA library. In contrast, a total of 22,506 reads aligned to Balamuthia (Fig. 5a and 5b), of which 20,145 were taxonomically assigned to Balamuthia mandrillaris (Additional file 1: Table S1). These 20,145 reads comprised $79 \%$ of all species-level non-chordate (lacking a backbone) eukaryotic reads in the HD 6 CSF dataset, and mapped to available $16 \mathrm{~S}$ and $18 \mathrm{~S}$ sequences of Balamuthia mandrillaris in the NCBI nt reference database [38]. A minority of the non-chordate eukaryotic reads aligned to Acathamoeba spp. (145 reads). Reads to Balamuthia were also detected in the DNA (13 reads) and brain biopsy RNA libraries (9 reads). The coverage of the 16S rRNA gene in the RNA library was sufficiently high to assemble a 1,405 bp full-length contig sharing 99.9 \% identity with the 2046 strain of Balamuthia. In the $18 \mathrm{~S}$ locus, mapped NGS reads from the patient spanned $98.1 \%$ of the gene and were $99.1 \%$ identical by nucleotide. No NGS hits were detected to the RNAse P gene, the only additional Balamuthia gene represented in the NCBI nt reference database as of August 2015.

We then sought to determine in retrospect whether earlier detection and diagnosis of Balamuthia infection in the case patient by NGS would have been feasible. Metagenomic NGS of a day 1 CSF sample followed by SURPI analysis using the June 2014 NCBI nt reference database generated no sequence hits to Balamuthia (Fig. 5b; Additional file 1: Table S1). However, repeating the analysis after adding the draft genome sequence of Balamuthia mandrillaris to the reference database resulted in the detection of additional Balamuthia reads (Fig. 5b and c) Importantly, nine species-specific DNA reads were detected from day 1 CSF (Fig. 5b, red text; Table 3). Although only two of nine putative Balamuthia reads had identifiable translated nucleotide identity to any protein in the NCBI nr database, one of those reads was found to share $77 \%$ amino acid identity to the gluathione transferase protein from
Acanthamoeba castellani, and hence most likely represented a bona fide hit to Balamuthia. These findings also indicated that the detection of Balamuthia reads was not due to errors in the draft genome assembly from incorporation of contaminating sequences from other organisms. Thus, detection of Balamuthia from the patient's day 1 sample and a more timely diagnosis by metagenomic NGS would presumably not have been made without the availability of the full draft genome as part of the reference database used for alignment.

\section{Discussion}

In this study, we describe a 'virtuous cycle' of clinical sequencing in which the continually increasing breadth of microbial sequences in reference databases improves the sensitivity and accuracy of infectious disease diagnosis, in turn driving the sequencing of additional reference strains. The assembly of the first draft reference genome for Balamuthia not only enhances the potential sensitivity of metagenomic NGS for detecting this pathogen, as shown here, but also provides new target sequences such as the rps3 intron / intergenic region and 28S rRNA gene that can be leveraged for the future development of more sensitive and specific diagnostic assays. Given the lack of proven efficacious treatments for Balamuthia encephalitis, it is unclear whether even earlier diagnosis at HD 1 would have impacted the fulminant course of our case patient's infection. However, it has been suggested that timely intervention in cases of Balamuthia might lead to improved outcome [43]. In addition, promising new experimental treatments such as miltefosine $[3,9,41]$, administered to the survivor infected by the sequenced 2046 strain (Vollmer and Glaser, under review), are now available.

Unbiased metagenomic NGS is a powerful approach for diagnosis of infectious disease because it does not rely on the use of targeted primers and probes, but rather, detects any and all pathogens on the basis of uniquely identifying sequence information [44]. Rapid and accurate bioinformatics algorithms [21, 45-47] and computational pipelines [17] have also been developed, with the capacity to analyze metagenomic NGS data in clinically actionable time frames. We also demonstrate here the critical role of comprehensive reference genomes in the NGS diagnostic paradigm. The availability of pathogen genomes with coverage of all clinically relevant 


\section{A}
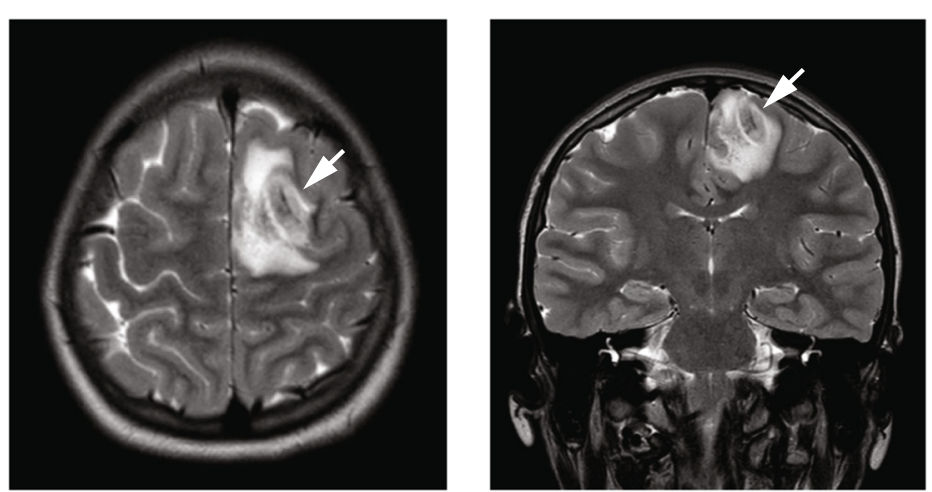

B
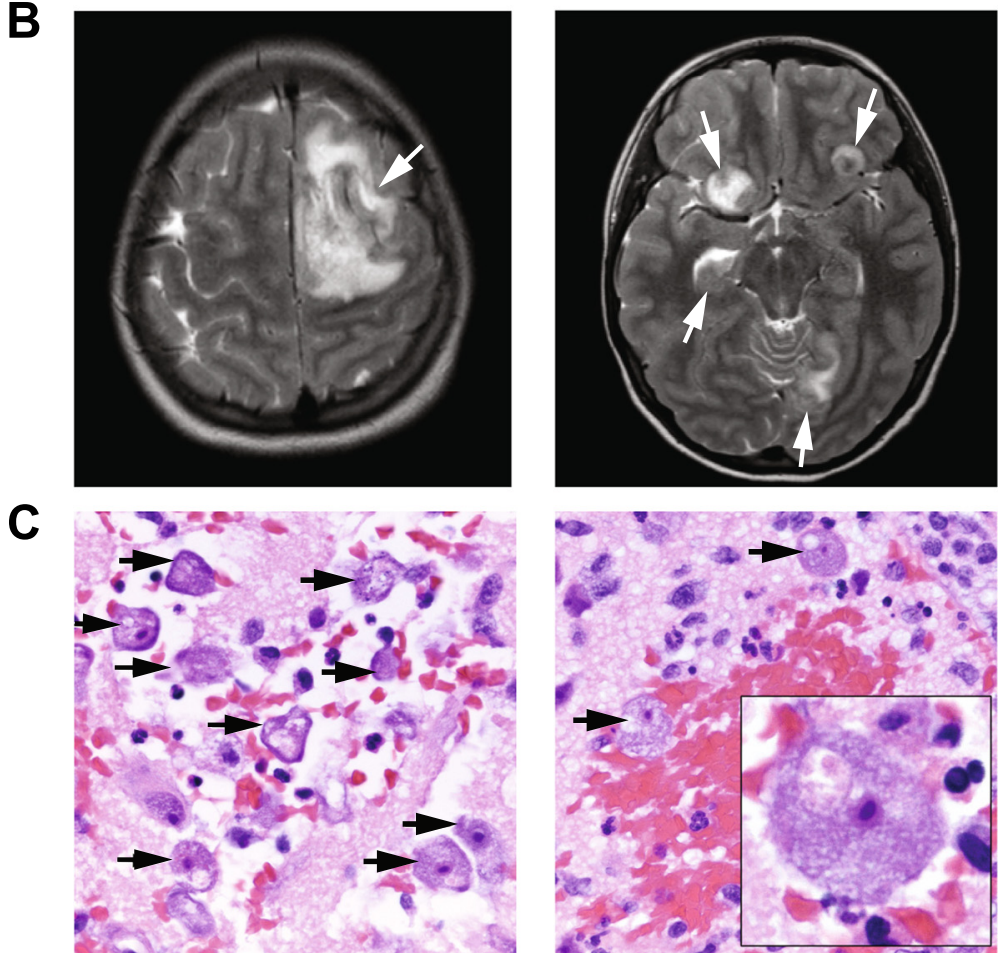

Fig. $4 \mathrm{MRI}$ and histopathology from a 15-year-old patient with a fulminant acute encephalitis. a A hospital day (HD) 1 coronal T2-weighted MR image, demonstrating a hemorrhagic lesion with surrounding edema within the superior left frontal lobe (left panel, white arrow) and left occipital lobe (right panel, white arrow). b A HD 5 contrast-enhanced T1-weighted MR image, revealing enlargement of the pre-existing left frontal lobe lesion (left panel, white arrow), as well as interval development of numerous additional rim-enhancing lesions in multiple regions (right panel, white arrows). c 20x (left and right panels) and 100x fields of view (right panel, inset) of a brain biopsy specimen from the patient demonstrating numerous viable, large amoebae (black arrows), with abundant basophilic vacuolated cytoplasm, round central nuclei, and prominent nucleoli, consistent with Balamuthia mandrillaris. There were areas of extensive hemorrhagic necrosis accompanied by a polymorphic inflammatory cell infiltrate including neutrophils and eosinophils (right panel)

genotypes can maximize the utility of NGS for not only diagnosis of individual patients $[15,16]$, but also public health applications such as transmission dynamics [48] and outbreak investigation [20,49]. Current limitations of metagenomic NGS for routine infectious disease diagnosis include: (1) the need to analyze millions of sequences in clinically actionable timeframes of minutes to hours (which has been at least partially addressed by rapid computational pipelines such as SURPI) [17]; (2) challenges in discriminating true pathogens from host flora or laboratory reagent contaminants in NGS data $[50,51]$; and (3) regulatory issues concerning validation of an assay targeting a potentially unlimited number of pathogens.

However, a key advantage of metagenomic NGS is that it does not rely on a priori suspicion of the etiology of the infectious agent. In our case patient, PAM was not clinically suspected, so specific PCR testing for Balamuthia was not done. Similarly, in another reported case of a women with endophthalmitis followed by meningoencephalitis [52], PAM also was not clinically suspected, requiring 


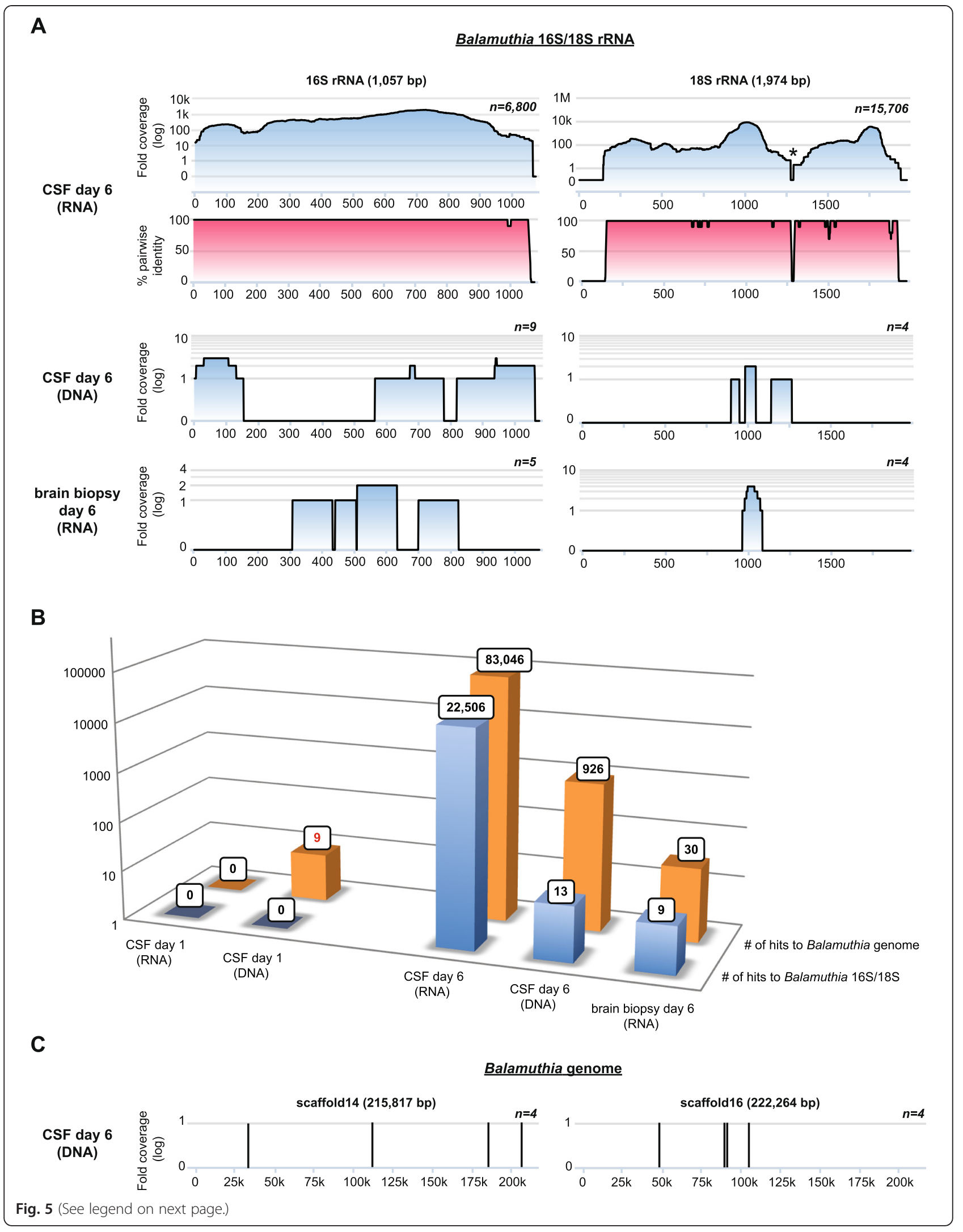


(See figure on previous page.)

Fig. 5 Identification of Balamuthia mandrillaris infection by metagenomic next-generation sequencing (NGS). a Coverage maps (blue gradient) and pairwise identity plots (magenta gradient) of two of the three available sequences from Balamuthia (16S/18S rRNA genes) in the NCBI nt reference database as of August 2015. Shown are coverage maps corresponding to day 6 DNA and RNA libraries from CSF and a day 6 mRNA library from brain biopsy. No hits to $16 \mathrm{~S}$ and $18 \mathrm{~S}$ Balamuthia sequences were seen from day 1 samples. The asterisk denotes an area with artificially low coverage after taxonomic classification of the NGS reads due to high conservation among eukaryotic sequences (for example, human, Balamuthia, and so on) within that region. b A bar graph of the number of species-specific NGS reads aligning to Balamuthia 16S/18S rRNA (blue) or the Balamuthia genome (orange) in day 1 or day 6 samples. Note that with the availability of the newly assembled 44 Mb Balamuthia genome, diagnosis of Balamuthia mandrillaris encephalitis at day 1 would have possible by detection of nine species-specific reads (red boldface). c Coverage maps of two large scaffolds, approximately $216 \mathrm{kB}$ and $222 \mathrm{kB}$ in size, from the Balamuthia draft genome, showing eight out of 926 hits to Balamuthia in the day 6 CSF DNA library that are identified by SURPI after the draft genome sequence is added to the reference database (versus only 13 hits previously)

metagenomic NGS for diagnosis. Although broad-range PCR assays targeting the conserved bacterial $16 \mathrm{~S}$ rRNA gene and eukaryotic 18S rRNA gene are available in select reference laboratories, there have been some concerns raised regarding sensitivity [53]. In addition, it is highly unlikely that $18 \mathrm{~S}$ PCR sequencing would have been positive in the patient's day 1 CSF given the absence of Balamuthia $18 \mathrm{~S}$ sequences in the case patient's metagenomic NGS data (Fig. 5b).

In the field of amoebic encephalitis, draft genomes are now available for Acanthamoeba castellani [32], Naegleria fowleri [54], and Balamuthia mandrillaris. However, more sequencing is certainly necessary to better understand the genetic diversity of these eukaryotic pathogens. In particular, shotgun sequencing and comparative analysis of mitochondrial genomes from seven Balamuthia strains uncovered at least three unique lineages, one of which was comprised entirely of amoebae isolated from California, revealing that geographic differences likely exist among strains (Fig. 1b). This study also identified a unique locus in a putative rps3 intron / intergenic region of the mitochondrial genome that is an attractive target for a clinical genotyping assay
(Figs. 1c and 2). Given the rarity of the disease, it is unknown whether infection by different strains of Balamuthia would affect clinical course or outcome, although the future availability of routine genotyping assays could help in addressing this question.

Limitations to this study include the small number of accessible clinical samples of Balamuthia mandrillaris infection and assembly of a draft genome with $>14,000$ scaffolds as a result of restricting the sequencing to short reads. Notably, an independently assembled draft genome of Balamuthia mandrillaris, comprising only 1,605 total contigs as a result of leveraging both long-read (PacBio) and short read (Illumina) technologies, has recently been published [39]. Additional messenger RNA (mRNA) sequencing of Balamuthia will still be needed to predict transcripts, identify splice junctions, and facilitate complete annotation of the genome.

\section{Conclusions}

We demonstrate here that the availability of pathogen reference genomes is critical for the sensitivity and success of unbiased metagenomic next-generation sequencing approaches in diagnosing infectious disease. In hindsight,

Table 3 Balamuthia mandrillaris reads from the day 1 patient sample

\begin{tabular}{|c|c|c|c|c|c|c|}
\hline $\begin{array}{l}\text { Read } \\
\text { no. }\end{array}$ & $\begin{array}{l}\text { Top NCBI NR match } \\
\text { by BLASTx }\end{array}$ & $\begin{array}{l}\% \text { amino } \\
\text { acid identity }\end{array}$ & $\begin{array}{l}\text { E- } \\
\text { value }\end{array}$ & $\begin{array}{l}\text { Matched scaffold } \\
\text { in Balamuthia genome }\end{array}$ & $\begin{array}{l}\text { \% nucleotide identity } \\
\text { (Balamuthia genome) }\end{array}$ & $\begin{array}{l}\text { E-value } \\
\text { (Balamuthia genome) }\end{array}$ \\
\hline 1 & None & - & - & scaffold46 & $96 \%$ & $5.00 \mathrm{E}-29$ \\
\hline 2 & None & - & - & scaffold26764 & $96 \%$ & $3.00 \mathrm{E}-27$ \\
\hline 3 & None & - & - & scaffold45 & $100 \%$ & $3.00 \mathrm{E}-36$ \\
\hline 4 & None & - & - & scaffold353 & $98 \%$ & $2.00 \mathrm{E}-24$ \\
\hline 5 & $\begin{array}{l}\text { Fumareylacetoacetase } \\
\text { (Flavobacteriales bacterium BRH_c54) }\end{array}$ & $82 \%$ & $\begin{array}{l}3.00 \mathrm{E}- \\
05\end{array}$ & scaffold203 & $100 \%$ & $3.00 \mathrm{E}-36$ \\
\hline 6 & None & - & - & scaffold106 & $96 \%$ & $1.00 \mathrm{E}-26$ \\
\hline 7 & $\begin{array}{l}\text { Glutathione transferase protein } \\
\text { (Acanthoamoeba castellani str. Neff) }\end{array}$ & $77 \%$ & 0.022 & scaffold30 & $99 \%$ & $8.00 \mathrm{E}-34$ \\
\hline 8 & None & - & - & scaffold112 & $97 \%$ & $7.00 \mathrm{E}-28$ \\
\hline 9 & None & - & - & scaffold511 & $98 \%$ & $5.00 \mathrm{E}-26$ \\
\hline
\end{tabular}


more timely and potentially actionable diagnosis at hospital day 1 in a fatal case of PAM from Balamuthia mandrillaris would have required the availability of the full genome sequence. Thus, in addition to revealing a significant amount of evolutionary diversity, the draft genome of Balamuthia mandrillaris presented here will improve the sensitivity of sequencing-based efforts for diagnosis and surveillance, and can be used to guide the development of targeted assays for genotyping and detection. The draft genome also constitutes a valuable resource for future studies investigating the biology of this eukaryotic pathogen and its etiologic role in PAM.

\section{Additional file}

Additional file 1: Table S1. SURPI clinical metagenomic results (XLSX $390 \mathrm{~kb})$

\section{Competing interests}

CYC is the director of the UCSF-Abbott Viral Diagnostics and Discovery Center (VDDC) and receives research support in pathogen discovery from Abbott Laboratories, Inc. The other authors declare that they have no competing interests.

\section{Authors' contributions}

$A L G, K M, S D$, and CYC conceived of and designed the study. ALG, TD, JB, and SY performed the experiments. KM, DM, YN, MV, CAP, BKK, and SR took care of patients with Balamuthia infection and contributed clinical samples. KM, $\mathrm{DM}, \mathrm{CG}, \mathrm{MV}, \mathrm{CAP}, \mathrm{BKK}, \mathrm{SR}$, and CYC analyzed the clinical and epidemiological data. ALG, SNN, SF, JB, and CYC analyzed the genomic sequencing data. ALG, SNN, SF, and CYC developed and contributed software analysis tools. ALG, KM, and $\mathrm{CYC}$ wrote the manuscript. All authors read and approved final manuscript.

\section{Acknowledgements}

This study was supported by grants from the National Institutes of Health (NIH) R01-HL105704 (to CYC) and UL1-TR001082 (to KM and SD), the Centers for Disease Control and Prevention (CDC) Emerging Infections Program U50/ CCU915546-09 (CG), University of California Discovery Grants Award \#180966 from the UC Office of the President, and an Abbott Pathogen Discovery Award (CYC).

\section{Author details \\ ${ }^{1}$ Department of Laboratory Medicine, University of California, 185 Berry Street, Box 0134, San Francisco, CA 94107, USA. ${ }^{2}$ UCSF-Abbott Viral Diagnostics and Discovery Center, San Francisco, CA 91407, USA. ${ }^{3}$ Children's Hospital Colorado and University of Colorado School of Medicine, Aurora, CO, USA. ${ }^{4}$ California Department of Public Health, Richmond, CA, USA. ${ }^{5}$ Kaiser Permanente Hospital, Oakland, CA, USA. ${ }^{6}$ John Muir Hospital, Walnut Creek, CA, USA. ${ }^{7}$ Department of Medicine, Division of Infectious Diseases, University of California, San Francisco, CA 94107, USA.}

Received: 18 August 2015 Accepted: 13 October 2015

Published online: 01 December 2015

\section{References}

1. Visvesvara GS. Epidemiology of infections with free-living amebas and laboratory diagnosis of microsporidiosis. Mt Sinai J Med. 1993;60:283-8.

2. Matin A, Siddiqui $R$, Jayasekera $S$, Khan NA. Increasing importance of Balamuthia mandrillaris. Clin Microbiol Rev. 2008;21:435-48.

3. Schuster FL, Guglielmo BJ, Visvesvara GS. In-vitro activity of miltefosine and voriconazole on clinical isolates of free-living amebas: Balamuthia mandrillaris, Acanthamoeba spp., and Naegleria fowleri. J Eukaryot Microbiol. 2006;53:121-6.
4. Visvesvara GS, Martinez AJ, Schuster FL, Leitch GJ, Wallace SV, Sawyer TK, et al. Leptomyxid ameba, a new agent of amebic meningoencephalitis in humans and animals. J Clin Microbiol. 1990;28:2750-6.

5. Schuster FL, Dunnebacke TH, Booton GC, Yagi S, Kohlmeier CK, Glaser C, et al. Environmental isolation of Balamuthia mandrillaris associated with a case of amebic encephalitis. J Clin Microbiol. 2003;41:3175-80.

6. Ahmad AF, Heaselgrave W, Andrew PW, Kilvington S. The in vitro efficacy of antimicrobial agents against the pathogenic free-living amoeba Balamuthia mandrillaris. J Eukaryot Microbiol. 2013;60:539-43.

7. Deetz TR, Sawyer MH, Billman G, Schuster FL, Visvesvara GS. Successful treatment of Balamuthia amoebic encephalitis: presentation of 2 cases. Clin Infect Dis. 2003;37:1304-12.

8. Kato H, Mitake S, Yuasa H, Hayashi S, Hara T, Matsukawa N. Successful treatment of granulomatous amoebic encephalitis with combination antimicrobial therapy. Intern Med. 2013;52:1977-81.

9. Martinez DY, Seas C, Bravo F, Legua P, Ramos C, Cabello AM, et al. Successful treatment of Balamuthia mandrillaris amoebic infection with extensive neurological and cutaneous involvement. Clin Infect Dis. 2010;51:e7-e11.

10. Schuster FL, Visvesvara GS. Axenic growth and drug sensitivity studies of Balamuthia mandrillaris, an agent of amebic meningoencephalitis in humans and other animals. J Clin Microbiol. 1996;34:385-8.

11. Qvarnstrom Y, Visvesvara GS, Sriram R, da Silva AJ. Multiplex real-time PCR assay for simultaneous detection of Acanthamoeba spp., Balamuthia mandrillaris, and Naegleria fowleri. J Clin Microbiol. 2006;44:3589-95.

12. Yagi S, Booton GC, Visvesvara GS, Schuster FL. Detection of Balamuthia mitochondrial 16S rRNA gene DNA in clinical specimens by PCR. J Clin Microbiol. 2005:43:3192-7.

13. Perez MT, Bush LM. Fatal amebic encephalitis caused by Balamuthia mandrillaris in an immunocompetent host: a clinicopathological review of pathogenic freeliving amebae in human hosts. Ann Diagn Pathol. 2007;11:440-7.

14. Schuster FL, Glaser C, Honarmand S, Maguire JH, Visvesvara GS. Balamuthia amebic encephalitis risk, Hispanic Americans. Emerg Infect Dis. 2004;10:1510-2.

15. Naccache SN, Peggs KS, Mattes FM, Phadke R, Garson JA, Grant P, et al. Diagnosis of neuroinvasive astrovirus infection in an immunocompromised adult with encephalitis by unbiased next-generation sequencing. Clin Infect Dis. 2015;60:919-23.

16. Wilson MR, Naccache SN, Samayoa E, Biagtan M, Bashir H, Yu G, et al. Actionable diagnosis of neuroleptospirosis by next-generation sequencing. N Engl J Med. 2014;370:2408-17.

17. Naccache SN, Federman S, Veeraraghavan N, Zaharia M, Lee D, Samayoa E, et al. A cloud-compatible bioinformatics pipeline for ultrarapid pathogen identification from next-generation sequencing of clinical samples. Genome Res. 2014:24:1180-92.

18. Schubert RD, Wilson MR. A tale of two approaches: how metagenomics and proteomics are shaping the future of encephalitis diagnostics. Curr Opin Neurol. 2015;28:283-7.

19. Fricke WF, Rasko DA. Bacterial genome sequencing in the clinic bioinformatic challenges and solutions. Nat Rev Genet. 2014;15:49-55.

20. Greninger AL, Naccache SN, Messacar K, Clayton A, Yu G, Somasekar S, et al. A novel outbreak enterovirus D68 strain associated with acute flaccid myelitis cases in the USA (2012-14): a retrospective cohort study. Lancet Infect Dis. 2015;15:671-82.

21. Zaharia M, Bolosky W, Curtis K, Fox A, Patterson D, Shenker S, et al. Faster and more accurate sequence alignment with SNAP. arXivorg. 2011;1111:5572.

22. Lares-Jimenez LF, Gamez-Gutierrez RA, Lares-Villa F. Novel culture medium for the axenic growth of Balamuthia mandrillaris. Diagn Microbiol Infect Dis. 2015:82:286-8

23. O'Connell J, Schulz-Trieglaff O, Carlson E, Hims MM, Gormley NA, Cox AJ. NxTrim: optimized trimming of Illumina mate pair reads. Bioinformatics. 2015;31:2035-7.

24. Bankevich A, Nurk S, Antipov D, Gurevich AA, Dvorkin M, Kulikov AS, et al. SPAdes: a new genome assembly algorithm and its applications to singlecell sequencing. J Comput Biol. 2012;19:455-77.

25. Greninger AL, Chorny I, Knowles S, Ng VL, Chaturvedi V. Draft genome sequences of four NDM-1-Producing Klebsiella pneumoniae strains from a health care facility in northern California. Genome Announc. 2015:3:3.

26. Lagesen $\mathrm{K}$, Hallin P, Rodland EA, Staerfeldt HH, Rognes T, Ussery DW. RNAmmer: consistent and rapid annotation of ribosomal RNA genes. Nucleic Acids Res. 2007:35:3100-8.

27. Lowe TM, Eddy SR. tRNAscan-SE: a program for improved detection of transfer RNA genes in genomic sequence. Nucleic Acids Res. 1997;25:955-64. 
28. Altschul SF, Gish W, Miller W, Myers EW, Lipman DJ. Basic local alignment search tool. J Mol Biol. 1990;215:403-10.

29. Soding J, Biegert A, Lupas AN. The HHpred interactive server for protein homology detection and structure prediction. Nucleic Acids Res. 2005;33:W244-8.

30. Martin M. Cutadapt removes adapter sequences from high-throughput sequencing reads. EMBnet J. 2011;17:1.

31. Kaijtani R, Toshimoto K, Noguchi H, Toyoda A, Ogura Y, Okuno M, et al. Efficient de novo assembly of highly heterozygous genomes from wholegenome shotgun short reads. Genome Res. 2014;24:1384-95.

32. Burger G, Plante I, Lonergan KM, Gray MW. The mitochondrial DNA of the amoeboid protozoon, Acanthamoeba castellanii: complete sequence, gene content and genome organization. J Mol Biol. 1995;245:522-37.

33. Herman EK, Greninger AL, Visvesvara GS, Marciano-Cabral F, Dacks JB, Chiu $\mathrm{CY}$. The mitochondrial genome and a 60-kb nuclear DNA segment from Naegleria fowleri, the causative agent of primary amoebic meningoencephalitis. J Eukaryot Microbiol. 2013;60:179-91.

34. Fukami H, Chen CA, Chiou CY, Knowlton N. Novel group I introns encoding a putative homing endonuclease in the mitochondrial cox1 gene of Scleractinian corals. J Mol Evol. 2007;64:591-600.

35. Zheng Z, Jiang K, Huang C, Mei C, Han R. Cordyceps militaris (Hypocreales: Cordycipitaceae): transcriptional analysis and molecular characterization of cox1 and group I intron with putative LAGLIDADG endonuclease. World J Microbiol Biotechnol. 2012;28:371-80.

36. Laroche J, Bousquet J. Evolution of the mitochondrial rps3 intron in perennial and annual angiosperms and homology to nad5 intron 1. Mol Biol Evol. 1999;16:441-52.

37. Regina TM, Picardi E, Lopez L, Pesole G, Quagliariello C. A novel additional group II intron distinguishes the mitochondrial rps 3 gene in gymnosperms. J Mol Evol. 2005;60:196-206.

38. Booton GC, Carmichael JR, Visvesvara GS, Byers TJ, Fuerst PA. Genotyping of Balamuthia mandrillaris based on nuclear 185 and mitochondrial 165 rRNA genes. Am J Trop Med Hyg. 2003;68:65-9.

39. Detering H, Aebischer T, Dabrowski PW, Radonic A, Nitsche A, Renard BY, et al. First draft genome sequence of Balamuthia mandrillaris, the causative agent of amoebic encephalitis. Genome Announc. 2015;3:5.

40. Kordis D. A genomic perspective on the chromodomain-containing retrotransposons: Chromoviruses. Gene. 2005:347:161-73.

41. Centers for Disease C, Prevention. Investigational drug available directly from CDC for the treatment of infections with free-living amebae. MMWR Morb Mortal Wkly Rep. 2013;62:666.

42. Kiderlen AF, Radam E, Lewin A. Detection of Balamuthia mandrillaris DNA by real-time PCR targeting the RNase P gene. BMC Microbiol. 2008;8:210.

43. Bakardjiev A, Azimi PH, Ashouri N, Ascher DP, Janner D, Schuster FL, et al. Amebic encephalitis caused by Balamuthia mandrillaris: report of four cases. Pediatr Infect Dis J. 2003:22:447-53.

44. Chiu CY. Viral pathogen discovery. Curr Opin Microbiol. 2013;16:468-78.

45. Buchfink B, Xie C, Huson DH. Fast and sensitive protein alignment using DIAMOND. Nat Methods. 2015;12:59-60.

46. Freitas TA, Li PE, Scholz MB, Chain PS. Accurate read-based metagenome characterization using a hierarchical suite of unique signatures. Nucleic Acids Res. 2015;43:e69.

47. Wood DE, Salzberg SL. Kraken: ultrafast metagenomic sequence classification using exact alignments. Genome Biol. 2014;15:R46.

48. Grad YH, Lipsitch M. Epidemiologic data and pathogen genome sequences: a powerful synergy for public health. Genome Biol. 2014;15:538.

49. Briese T, Paweska JT, McMullan LK, Hutchison SK, Street C, Palacios G, et al. Genetic detection and characterization of Lujo virus, a new hemorrhagic feverassociated arenavirus from southern Africa. PLoS Pathog. 2009:5:e1000455.

50. Naccache SN, Greninger AL, Lee D, Coffey LL, Phan T, Rein-Weston A, et al. The perils of pathogen discovery: origin of a novel parvovirus-like hybrid genome traced to nucleic acid extraction spin columns. J Virol. 2013:87:11966-77.

51. Strong MJ, Xu G, Morici L, Splinter Bon-Durant S, Baddoo M, Lin Z, et al. Microbial contamination in next generation sequencing: implications for sequence-based analysis of clinical samples. PLoS Pathog. 2014;10:e1004437.

52. Wilson MR, Shanbhag NM, Reid MJ, Singhal NS, Gelfand JM, Sample HA, et al. Diagnosing Balamuthia mandrillaris encephalitis with metagenomic deep sequencing. Ann Neurol. 2015. doi: 10.1002/ ana.24499. [Epub ahead of print]
53. Petti CA. Detection and identification of microorganisms by gene amplification and sequencing. Clin Infect Dis. 2007:44:1108-14.

54. Zysset-Burri DC, Muller N, Beuret C, Heller M, Schurch N, Gottstein B, et al. Genome-wide identification of pathogenicity factors of the free-living amoeba Naegleria fowleri. BMC Genomics. 2014;15:496.

55. Bakardjiev A, Azimi PH, Ashouri N, Ascher DP, Janner D, Schuster FL, et al. Amebic. encephalitis caused by Balamuthia mandrillaris: report of four cases. Pediatr Infect Dis J 2003, 22:447-453.

56. Dunnebacke TH, Schuster FL, Yagi S, Booton GC. Balamuthia mandrillaris from soil samples. Microbiology. 2004, 150:2837-2842.

57. Gordon SM, Steinberg JP, DuPuis MH, Kozarsky PE, Nickerson JF, Visvesvara GS et al. Culture isolation of Acanthamoeba species and leptomyxid amebas from patients with amebic meningoencephalitis, including two patients with AIDS. Clin Infect Dis 1992, 15:1024-1030.

58. Yang $\mathrm{XH}$, Jones D, Kaeppen A, Qian J: Amebic meningoencephalitis in a 6 year-old girl caused by Balamuthia mandrsillaris. J Neuropathol Exp Neurol 2001, 60:556.

\section{Submit your next manuscript to BioMed Central and take full advantage of:}

- Convenient online submission

- Thorough peer review

- No space constraints or color figure charges

- Immediate publication on acceptance

- Inclusion in PubMed, CAS, Scopus and Google Scholar

- Research which is freely available for redistribution 